\title{
The role of FOXP3 of interstitial tissue in the pathogenesis of breast cancer
}

\author{
Yu-Juan Xiang ${ }^{1,2}$, Man Feng $^{3}$, Li-Yuan Liu ${ }^{1,2}$, Fei Wang ${ }^{1,2}$, Li-Xiang Yu ${ }^{1,2}$, Fei Zhou ${ }^{1,2}$, Shu-Ya Huang ${ }^{1,2}$, \\ Chao Zheng ${ }^{1,2}$, Chun-Miao Ye ${ }^{1,4}$, Wen-Zhong Zhou ${ }^{1,2}$, Geng-Shen Yin ${ }^{1,4}$, Zhong-Bing Ma ${ }^{1,2}$, \\ Liang Li ${ }^{1,2}$, Qiang Zhang ${ }^{1,2}$, Qin-Ye Fu ${ }^{1,2}$, Zhi-Gang Yu ${ }^{1,2}$
}

${ }^{1}$ Department of Breast Surgery, The Second Hospital, Cheeloo College of Medicine, Shandong University, Jinan, China; ${ }^{2}$ Institute of Translational Medicine of Breast Disease Prevention and Treatment, Shandong University, Jinan, China; ${ }^{3}$ Department of Pathology, The Third Affiliated Hospital of Shandong First Medical University (Affiliated Hospital of Shandong Academy of Medical Sciences), Shandong Academy of Medical Sciences, Jinan, China; ${ }^{4}$ School of Medicine, Shandong University, Jinan, China

Contributions: (I) Conception and design: YJ Xiang, QY Fu, ZG Yu; (II) Administrative support: ZG Yu; (III) Provision of study materials or patients: YJ Xiang, ZB Ma; (IV) Collection and assembly of data: YJ Xiang, M Feng, ZB Ma, LY Liu; (V) Data analysis and interpretation: YJ Xiang, LY Liu; (VI) Manuscript writing: All authors; (VII) Final approval of manuscript: All authors.

Correspondence to: Zhi-Gang Yu, PhD, MD; Qin-Ye Fu. Department of Breast Surgery, The Second Hospital, Cheeloo College of Medicine, Shandong University, 247 Beiyuan St., Jinan 250033, China. Email: yuzhigang@sdu.edu.cn; fqyhome@163.com.

Background: The study aimed to evaluate the expression level of FOXP3 and the degree of FOXP3 positive lymphocytes in the interstitial tissues of normal breast tissues, primary breast cancer tissues and axillary lymph node metastatic cancer tissues, so as to better clarify FOXP3's role in the process of breast cancer tumorigenesis and progression.

Methods: FOXP3 protein expression and FOXP3 positive tumor infiltrating lymphocytes were investigated by immunohistochemistry analysis in normal breast tissues, primary breast cancer tissues and axillary lymph node metastatic cancer tissues achieved from 44 patients.

Results: Compared to normal breast tissues, the expression of FOXP3 in interstitial tissues in primary and metastatic cancer tissues was significantly up-regulated. The FOXP3 positive lymphocytes infiltrated interstitial tissues in 30 of 44 (68.2\%) specimens of normal breast tissues, 40 of 44 (90.9\%) specimens of primary cancer tissues and 42 of $44(95.5 \%)$ specimens of axillary lymph node metastatic cancer tissues. There was linear correlation trend between FOXP3 expression and the process transforming from normal breast epithelium to primary cancer and metastatic cancer, indicating its potential role in the pathogenesis of this disease. And we found that the expression of interstitial FOXP3 in axillary lymph node metastatic cancer site was correlated with the lymph node ratio (LNR), and LNR was higher in the FOXP3 positive expression group than the FOXP3 absent group.

Conclusions: Increased FOXP3 expression in cancer interstitial tissues and FOXP3 positive lymphocytes infiltration might have a certain correlation with the development of breast cancer through facilitating immune escape of tumor.

Keywords: Breast cancer; FOXP3; lymph node ratio (LNR)

Received: 11 January 2021; Accepted: 22 April 2021; Published: 30 April 2021.

doi: $10.21037 /$ tbcr-21-1

View this article at: http://dx.doi.org/10.21037/tbcr-21-1 


\section{Introduction}

Breast cancer is one of the most common malignant disease among women all over the world. As the latest GLOBOCAN data shows that there are 2.09 million new breast cancer cases diagnosed in 2018, accounting for $24 \%$ of all malignant tumors for females (1). In addition, although preventive approaches and treatments have greatly improved recent years, breast cancer is still the top killer cancer for women in less developed countries. Metastasis to lymph node remains the most influential prognostic factor in disease progression, and acts as one of the important survival predicting factors $(2,3)$. Recent studies have established that axillary lymph node ratio (ratio of positive over excised lymph nodes, LNR) predicted outcomes of breast cancer more accurately than the number of involved nodes (4-8). The presence of breast cancer cells in axillary lymph nodes is associated with the alteration of lymphocytes population (9). CD4+ T lymphocytes and CD8+ T lymphocytes are the main cells for immune surveillance $(10,11)$, while forkhead box P3positive (FOXP3+) regulatory $\mathrm{T}$ cells (Tregs) are essential for immune tolerance (11). And evidence has shown that increased number of FOXP3+ Tregs correlates with disease prognosis in breast cancer $(12,13)$.

FOXP3 is an important member of the forkhead transcription factor family (14). Although many studies have focused on discovering the expression of FOXP3 in non-T-regulatory cells (15), FOXP3 is still the most specific marker of regulatory $\mathrm{T}$ cells (Tregs), which is critical for their development and function. Loss of FOXP3 function leads to Tregs deficiency. Tregs are considered to suppress $\mathrm{T}$ cell immune response, and increased Tregs activity may be correlated with poor immune responses to tumor antigens and may induced immune dysfunction. In most solid tumors, Tregs' infiltration has been related to worse outcomes (16). FOXP3+ Treg may act as an important indicator for the biological behavior of cancer, because the increased number of FOXP3+ Tregs in tumor microenvironment could stimulate the negative regulation of host immune response to facilitate the invasion and metastasis of cancer cells (17-19). And it has been reported an important effect of FOXP3 gene on breast cancer development by analyzing human breast cancer samples. Moreover, FOXP3 expression within tumorinflitrating $\mathrm{T}$ cells has been demonstrated to occur early during tumorigenesis in breast at the preinvasive stage (20). However, Several researches indicated that higher FOXP3 + Tregs was associated with poor prognosis in breast cancer (21), while some did not find such association or the opposite (22). And there is no difference in number of FOXP3+ Tregs between sentinel lymph nodes positive and sentinel lymph nodes negative (23). However, there is few studies on the relationship between FOXP3 expression and NLR, which can more accurately predict the outcomes of breast cancer. In short, study on the significance of FOXP3 in this disease is still insufficient. These above findings may suggest the important role of FOXP3+ Tregs in breast cancer tumorigenesis, but the exact value of interstitial FOXP3 expression in the breast cancer development process from normal to primary and metastasis hasn't been revealed yet.

The aim of this study was to evaluate the expression level of FOXP3 in interstitial tissues in matched normal breast tissues, primary breast cancer tissues and axillary lymph node metastatic cancer tissues of 44 breast cancer patients to better clarify FOXP3's role in the process of breast cancer tumorigenesis and progression. In addition, we also investigated the relationship between interstitial FOXP3 expression and axillary lymph node status to illustrate the clinical significance of FOXP3 expression in the disease. We present the following article in accordance with the STROBE reporting checklist (available at http://dx.doi. org/10.21037/tbcr-21-1).

\section{Methods}

This study was conducted in accordance with the Declaration of Helsinki (as revised in 2013) and the Harmonized Tripartite Guideline for Good Clinical Practice from the International Conference on Harmonization. This study was approved by Institutional Review Board of the Second Hospital of Shandong University. Informed consent was obtained from all individual participants included in the study.

\section{Study population}

There were 44 breast cancer patients with lymph node invasion enrolled, confirmed pathologically in the Second Hospital of Shandong University. The eligibility criteria included histologically diagnosis of invasive cancer of breast, without any preoperative therapy, lymph node metastasis, female, having available breast cancer tissue samples and obtained informed consent. And the exclusion criteria were as follows: diagnosed with other malignant diseases; pregnancy; participation in other trials; received preoperative therapy; with bilateral tumors of breast; with 
Table 1 The characteristics of 44 patients with breast cancer

\begin{tabular}{|c|c|c|}
\hline Characteristics & No. of patients & $\%$ \\
\hline \multicolumn{3}{|l|}{ Age (years) } \\
\hline$<50$ & 18 & 40.9 \\
\hline$\geq 50$ & 26 & 59.1 \\
\hline \multicolumn{3}{|l|}{ Tumor side } \\
\hline Left & 17 & 38.6 \\
\hline Right & 27 & 61.4 \\
\hline \multicolumn{3}{|l|}{ Lymph node } \\
\hline $\mathrm{N} 1$ & 21 & 47.7 \\
\hline N2 & 16 & 36.4 \\
\hline N3 & 7 & 15.9 \\
\hline \multicolumn{3}{|l|}{ ER } \\
\hline Negative & 11 & 25.0 \\
\hline Positive & 33 & 75.0 \\
\hline \multicolumn{3}{|l|}{ PR } \\
\hline Negative & 13 & 29.5 \\
\hline Positive & 31 & 70.5 \\
\hline \multicolumn{3}{|l|}{ ER/PR. } \\
\hline Negative & 8 & 18.2 \\
\hline Positive & 36 & 81.8 \\
\hline \multicolumn{3}{|l|}{ Her-2 } \\
\hline Negative & 32 & 72.7 \\
\hline Positive & 12 & 27.3 \\
\hline
\end{tabular}

a breast cancer family history. The characteristics of the enrolled 44 patients were displayed in Table 1.

\section{Collections of tissue samples}

All cancer tissues and the adjacent unaffected normal breast tissues as well as metastatic cancer tissues were collected from patients with breast cancer who had undergone surgical resection prior to chemotherapy. These adjacent uninvolved breast tissues obtained from the region more than $5 \mathrm{~cm}$ away from cancer sites. And the metastatic cancer tissues referred to lymph node with invasion. All the tissues specimens were collected from the tissues blocks fixed by formalin and embedded with paraffin. Pathologists reevaluated all enrolled samples to verify the diagnosis, in addition to estimate the amount of cells. The study was approved by the Regional Committee for Medical and Health Research Ethics (REK). Clinical information of the patients was achieved under guidelines and with approval from the Institutional Review Board of the Second Hospital of Shandong University.

\section{Immunobistochemical analyses}

Achieved formalin-fixed paraffin-embedded tumor and normal adjacent specimens as well as axillary lymph node metastatic specimens from breast cancer patients were used for immunohistochemical analyses of FOXP3 expression. The level of FOXP3 was evaluated by Streptavidinperoxidase-biotin (SP) immunohistochemical method. The slides were placed in an oven at $65^{\circ} \mathrm{C}$ for 40 minutes and quickly transferred to xylene for dewaxing. Then the tissues were hydrated with gradient alcohols. Immersed the hydrated slides in EDTA repair solution working fluids (1:50, Zhongshan Biotechnology Company, Beijing, China), and placed in microwave oven, to repair on high heat for 15 minutes for antigen retrieval. Three percent hydrogen peroxide acting for 15 minutes was used to quench the activity of endogenous peroxidase, then normal serum 20 minutes for blocking nonspecific bindings. The sections were incubated with mouse antiFOXP3 antibody [236A/E7] (1:200, abcam, ab20034) overnight at $4{ }^{\circ} \mathrm{C}$, then the secondary antibody (SP reagent kit, Zhongshan Biotechnology Company, PV9000, Beijing, China). Reagent 1 from the detection kit acted for 50 minutes and then Reagent 2 for another 50 minutes at room temperature. Diaminobenzidine (Zhongshan Biotechnology Company, Beijing, China) was used for color development, lastly counterstained by hematoxylin. PBS replaced the mouse anti-FOXP3 antibody was conducted as the negative control.

ER, PR, Her-2 protein expression status in all breast cancer tissues were achieved from clinical records of patients under guidelines.

\section{Assessment of Immunobistochemical results}

Two pathologists reviewed and scored the stained slides independently. The assessment included the intensity of staining as well as the proportion of positive staining, and the total score was decided by combining both parts. In brief, each slide was inspected under microscope in its entirety. Firstly, the proportion score represented 

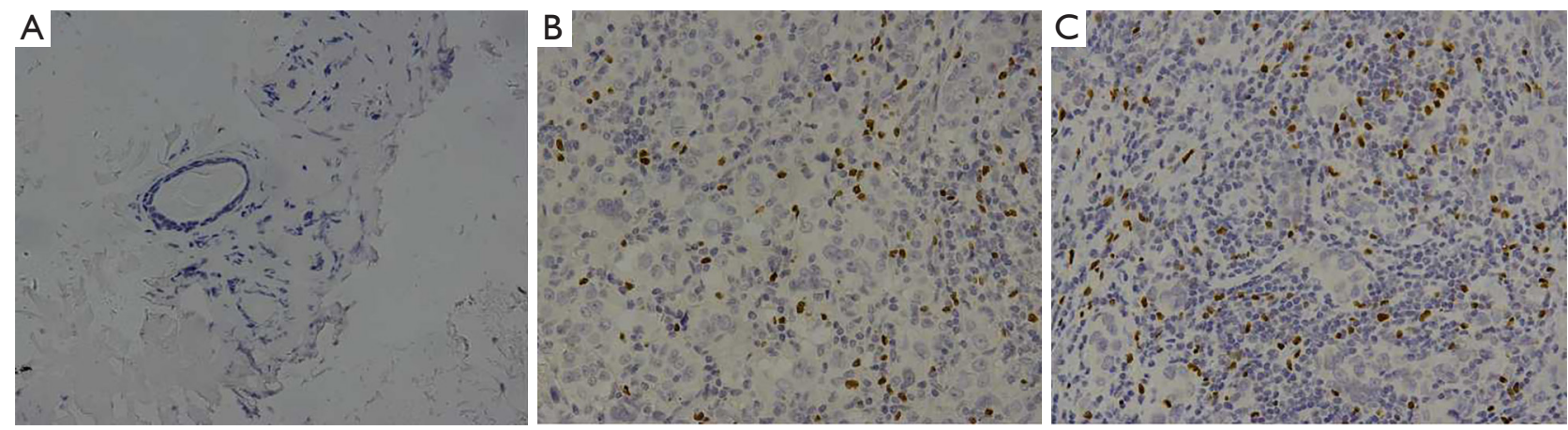

Figure 1 Representative immunohistochemical staining of intratumoral FOXP3 in breast tissues. (A-C) Positive expression of stromal FOXP3 in normal breast tissues, primary breast cancer and axillary lymph node metastatic cancer from same patient respectively (Original magnification, $\times 400$ ). The intensity as well as proportion score of FOXP3 staining was up-regulated in primary and metastatic cancer compared with the normal components.

the ratio of positive cells was assigned ( 0 , no positive cell; 1 , less than 1 positive cell among 100 cells; 2,1 to 10 positive cells among 100 cells; 3 , the positive ratio was between $1 / 10$ and $1 / 3 ; 4$, the positive ratio was between $1 / 3$ and $2 / 3$; and 5 , more than $2 / 3$ cells were positive). Then, the intensity score represented the average staining intensity of positive cells was assigned ( 0 , no staining; 1 , low-grade staining; 2 , moderate-grade staining; and 3, strong-grade staining). The total score was added by proportion and intensity score ranging from 0 to $8(24,25)$.

\section{Statistical analysis}

The statistical analysis was performed using the statistical software SPSS, version 16.0. Comparisons of FOXP3 expression (intensity, proportion and total score evaluated by the immuno-histochemistry) between primary breast cancer as well as metastatic cancer and noncancerous breast tissues were done using the Chisquare test for K-related samples. The association between FOXP3 expression in stromal cells and the normal epithelium-primary breast cancer-metastatic cancer process was evaluated by the Spearman's rho test, and its liner correlation was calculated by the Jonckheere-Terpstra test. And we investigated the correlation between FOXP3 expressed in breast interstitial tissues and lymph node status of patients by the Spearman's rho test and the Jonckheere-Terpstra test. All tests were two-sided and $\mathrm{P}<0.05$ was considered statistically significant.

\section{Results}

\section{The expression of FOXP3 was up-regulated significantly in primary and metastatic cancer tissues compared with its normal epithelial tissues}

The FOXP3 protein was distributed in the nucleus of interstitial lymphocytes (Figure 1). And as shown in Table 2, the FOXP3 + lymphocytes infiltrated interstitial tissues in 30 of $44(68.2 \%)$ specimens of normal breast tissues, 40 of 44 (90.9\%) specimens of primary cancer tissues and 42 of $44(95.5 \%)$ specimens of axillary lymph node metastatic cancer tissues, and the difference among three groups was statistically significant $\left(\chi^{2}=25.35, \mathrm{P}<0.001\right)$. In line with the different intensity of FOXP3 immunohistochemical staining, there were significant difference both in the proportion $\left(\chi^{2}=30.56, \mathrm{P}<0.001\right)$ and total score $\left(\chi^{2}=36.18\right.$, $\mathrm{P}<0.001)$ of FOXP3 expression among the three groups. Furthermore, we found that the intensity of interstitial FOXP3 in primary $(\mathrm{P}<0.001)$ as well as metastatic cancer $(\mathrm{P}<0.001)$ was increased significantly compared with their normal counterparts (Figure $2 A$ ). And the proportion of interstitial FOXP3 positive $\mathrm{T}$ cells in primary $(\mathrm{P}<0.001)$ and metastatic cancer $(\mathrm{P}=0.007)$ as well as the total score of interstitial FOXP3 positive $\mathrm{T}$ cells in primary $(\mathrm{P}<0.001)$ and metastatic cancer $(\mathrm{P}<0.001)$ was also increased significantly (Figure $2 B, C$ ). However, there was no significantly difference between primary cancer and metastatic cancer in present study. Therefore, the expression of FOXP3 in interstitial tissues was upregulated significantly in primary and metastatic cancer tissues compared with its normal interstitial tissues, 
Table 2 The expression of FOXP3 up-regulated significantly in primary and metastatic cancer interstitial tissues compared with its normal interstitial tissues

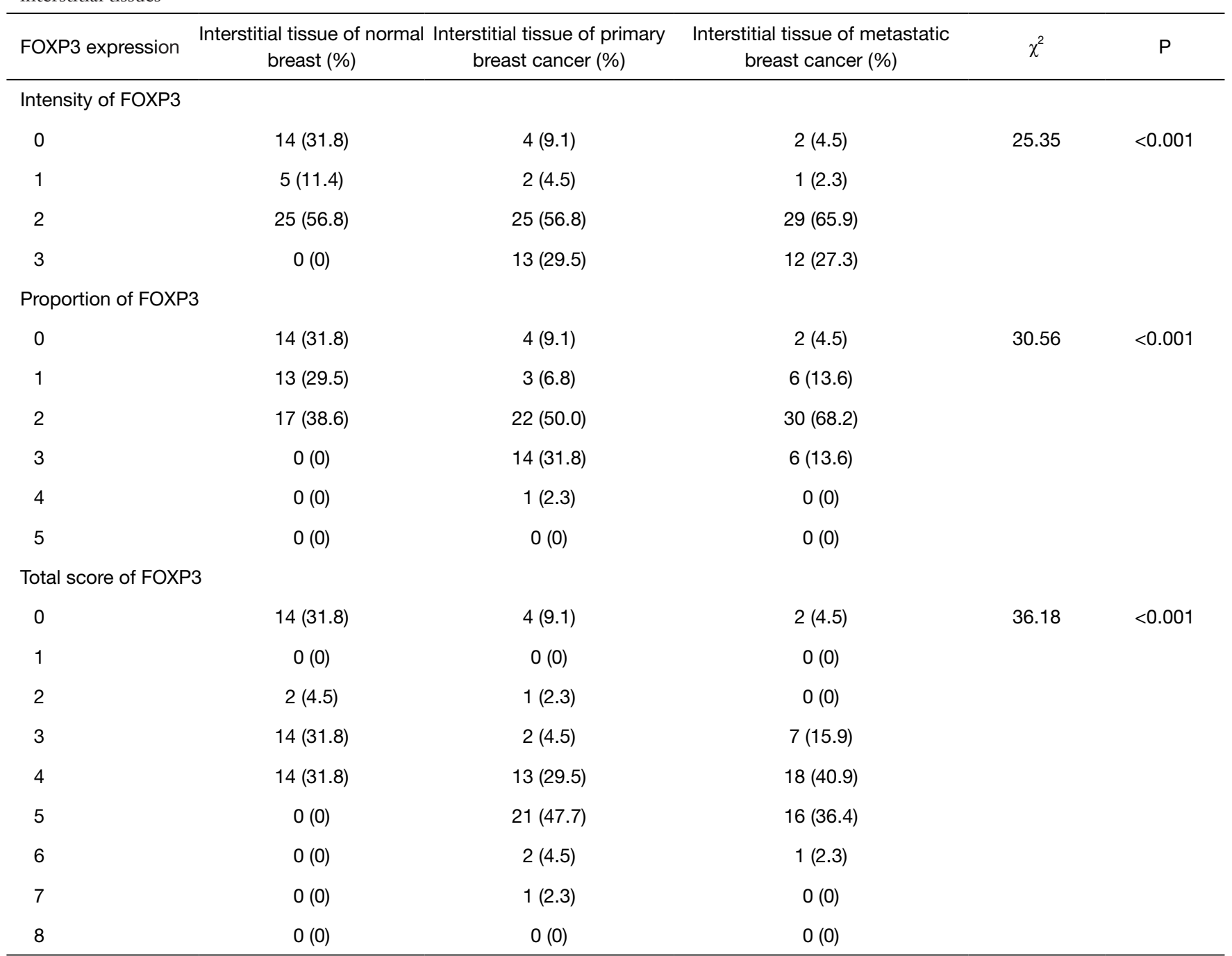

indicating its potential role in the development and progression of this disease.

\section{The linear correlation trend between FOXP3 expression and the process transforming from normal breast epithelium to primary cancer and metastatic cancer}

The correlation between FOXP3 expression in interstitial tissues and pathogenesis process from normal epithelium to primary and metastatic cancers was evaluated. As shown in Table 3, the result indicated that there was a significant positive correlation between expression intensity of FOXP3 in interstitial tissues and the pathogenesis stages of breast cancer $(r=0.412$, $\mathrm{P}<0.001)$. And the proportion of FOXP $3+$ lymphocytes $(r=0.366, P<0.001)$ as well as the total score of FOXP3 $(\mathrm{r}=0.442, \mathrm{P}<0.001)$ staining in interstitial tissues showed similar correlation with the pathogenesis stages. Additionally, the expression status of FOXP3 (including intensity, proportion and total score) and the pathogenesis process seemed to have a linear trend $(\mathrm{P}<0.001)$, which means the FOXP3 expression upregulated with the pathogenesis process transforming from normal epithelium to primary as well as metastatic cancers. The results indicated that FOXP3 involved in the pathogenesis of this disease. 

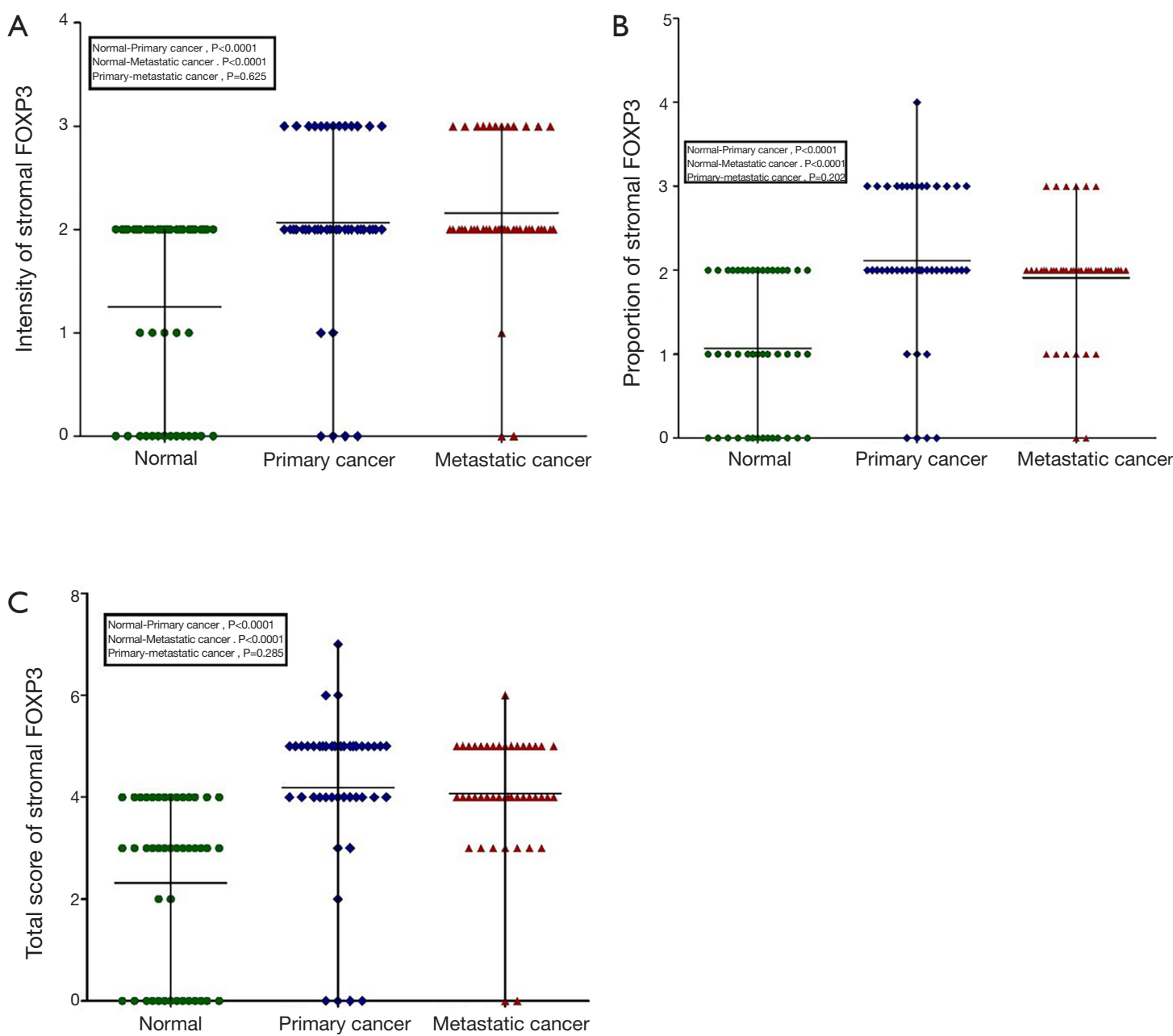

Figure 2 FOXP3 expression in normal, primary and metastatic breast cancer. (A) The intensity of interstitial FOXP3 expression in matched normal breast-primary breast cancer-axillary lymph node metastatic cancer. The intensity of interstitial FOXP3 increased significantly in primary and metastatic cancer compared to normal breast tissues, but no significant difference was found between the primary and metastatic cancer. Horizontal bars represent median values. (B) The proportion of interstitial FOXP3 expression in matched normal breastprimary breast cancer-axillary lymph node metastatic cancer. The proportion of interstitial FOXP3 increased significantly in primary and metastatic cancer compared to normal breast tissues, while no significant difference was found between the primary and metastatic cancer. Horizontal bars represent median values. (C) The total score of interstitial FOXP3 expression in matched normal breast-primary breast cancer-axillary lymph node metastatic cancer. The total score of interstitial FOXP3 increased significantly in primary and metastatic cancer compared to normal breast tissues, while no significant difference was found between the primary and metastatic cancer. Horizontal bars represent median values.

\section{The correlation between FOXP3 expressed in breast interstitial tissues and lymph node status of patients}

In order to investigate the potential role of FOXP3 expressed in interstitial tissues in breast cancer metastasis to lymph node, we evaluated FOXP3 expression status in primary breast cancer interstitial tissues and metastatic cancer interstitial tissues. The results showed that there was a significant positive correlation between the expression of FOXP3 (including intensity and proportion as well as total score) in interstitial tissues in metastatic axillary lymph nodes and the lymph node ratio (LNR). As shown in Table 4, the negative expression cases of FOXP3 seemed to present high LNR ( $\mathrm{r}=0.305, \mathrm{P}=0.044)$. However, no correlation between FOXP3 expressed in primary cancer interstitial 
Table 3 The linear correlation trend between the expression of FOXP3 and the process of normal breast epithelium-primary cancer-metastatic cancer

\begin{tabular}{lcccc}
\hline \multirow{2}{*}{ Expression of FOXP3 } & \multicolumn{4}{c}{ Normal breast epithelium-primary cancer-metastatic cancer } \\
\cline { 2 - 5 } & Correlation coefficient( $\mathrm{r}$ ) & $\mathrm{P}$ & J-T statistic & $\mathrm{p}$ \\
\hline Intensity of FOXP3 & 0.412 & $<0.001$ & 3888 & $<0.001$ \\
Proportion of FOXP3 & 0.366 & $<0.001$ & 3789 & $<0.001$ \\
Total score of FOXP3 & 0.442 & $<0.001$ & 4034 & $<0.001$ \\
\hline
\end{tabular}

Table 4 The linear correlation trend between the expression of FOXP3 and the lymph node ratio (LNR)

\begin{tabular}{lccc}
\hline \multirow{2}{*}{ Expression of FOXP3 } & & LNR & J-T statistic \\
\cline { 2 - 4 } & Correlation coefficient $(r)$ & $P$ & 0.644 \\
\hline Primary cancer stroma & 0.072 & 0.044 & 78 \\
Metastatic cancer stroma & 0.305 & 0.035 \\
\hline
\end{tabular}

tissues and lymph node status had been found in this study. The results above indicated that the expression of FOXP3 may be a potential marker for the LNR.

\section{Discussion}

FOXP3 has been widely accepted as one of the best markers for Tregs. Although many studies have focused on discovering the expression of FOXP3 in non-T-regulatory cells, such as cancer cells and macrophages, FOXP3 is still the most specific marker of regulatory $\mathrm{T}$ cells (Tregs), which is critical for their development and function. Loss of FOXP3 function leads to Tregs deficiency. Tumor-infiltrating FOXP3 positive Tregs were reported to be up-regulated in various tumors (26-29). And previous studies had indicated that CD4+CD25+FOXP3+ Tregs played a central role in tumor immune escape, such as lung cancer, melanoma and liver cancer (30,31). FOXP3 showed critical roles in controlling local immune response and promoting the immune escape of tumors. In this study, we found that FOXP3+ lymphocytes infiltrated interstitial tissues in 30 of $44(68.2 \%)$ specimens of normal breast tissues, 40 of 44 (90.9\%) specimens of primary cancer tissues and 42 of 44 (95.5\%) specimens of axillary lymph node metastatic cancer tissues, and the difference among three groups was statistically significant. In line with the different expression intensity of FOXP3, there were significant difference both in the proportion and total score of FOXP3 expression among the three groups. What's more, we also found a liner correlation trend between FOXP3 expressed in interstitial tissues and pathogenesis process from normal epithelium to primary and metastatic cancer, meant that FOXP3 expression and number of FOXP3 positive lymphocytes up-regulated with the pathogenesis process transforming from normal epithelium to primary as well as metastatic cancers. Researches had consistently identified that the number of FOXP3 + Tregs increased in breast cancer $(32,33)$. Thus, these increased FOXP3 expression in tumor microenvironment may be the reason for negative regulation of anti-cancer immune response to promote tumor formation and metastasis. Our results above also suggested that FOXP3 was involved in the pathogenesis of breast cancer.

Previous studies had found that infiltration of Tregs occurred as an early event during the tumorigenesis of breast cancer, for Tregs infiltration up-regulated between normal and DCIS components as well as between DCIS and IDC lesions significantly (20). However, it was still unclear whether FOXP3 + Tregs participated both in breast tumor formation and progression. In our present study, we investigated the variation of FOXP3 expression in the pathogenesis process from normal to axillary lymph node metastasis, to figure out which stage it played an important role in. We noted a significantly up-regulation of interstitial FOXP3 expression in primary and metastatic cancer tissues compared to its normal components, revealing its potential effect in the development and progression of this disease. However, we didn't find an obvious change between primary breast cancer and lymph nodes metastatic cancer in FOXP3 expression. Thus, our results clearly demonstrated that the 
up-regulation of FOXP3 expression was essential for the tumor formation, which however was not necessary for the metastasis to axillary lymph nodes. Our results demonstrated the previous hypothesis that Treg-mediated immune escape might be a pivotal early requirement for tumor progression in breast cancer. These findings were also in accordance with Yesim's observation that there's no significant difference in number of FOXP3 + Tregs between sentinel node positive and sentinel node negative samples (23), for the regulation of FOXP3 expression was occurred early in the tumorigenesis in breast cancer. However, further studies are needed to confirm this hypothesis in future.

The axillary lymph node status was one of the most powerful prognosis predictor for breast cancer (34). Recent studies had established that axillary lymph node ratio (ratio of positive over excised lymph nodes, LNR) predicted outcomes of breast cancer more accurately than the number of involved nodes (4-8). In the study here, we found that the expression of interstitial FOXP3 in axillary lymph node metastatic cancer site was correlated with the LNR, and LNR was higher in the positive FOXP3 expression group than the FOXP3 absent group. Although we hadn't found significant correlation between interstitial FOXP3 expression in primary breast cancer and LNR, the result still provided a potential marker for the LNR. However, the results need to be further tested, because of the limited sample size of present study.

Our study still had several limitations. First, the sample size was relatively small, the results might be biased to some degree. Second, we examined the whole nuclear expression level of FOXP3 without distinguishing its specific isoforms or subcellular locations, for some studies recently reported that there were multiple isoforms of FOXP3 and different isoforms located in different subcellular sites might display variable influence on the development and function of Tregs (35). Further studies on the isoforms and subcellular locations may provide more in understanding the true function of FOXP3 in cancers. Despite the above limitations, the study is still meaningful for it has revealed the exact development process of breast cancer in which FOXP3 plays roles.

\section{Conclusions}

In summary, we found that the expression of interstitial FOXP3 in primary breast cancer as well as lymph node metastatic cancer were increased significantly compared with normal breast tissues, but there was no obvious difference between primary and metastatic cancer, which supported the hypothesis that Treg-mediated immune escape may be a pivotal early requirement for tumor progression in breast cancer. What's more, we identified that the FOXP3 may be a potential marker for the LNR in breast cancer. In conclusion, increased FOXP3 expression in cancer interstitial tissues and FOXP3 positive lymphocytes infiltration might be closely related to the tumorigenesis and progression of breast cancer by promoting immune escape of the tumor.

\section{Acknowledgments}

Funding: This research was primarily funded by the Major Scientific and Technological Innovation Project of Shandong Province (2017CXGC1212), the National Natural Science Foundation of China (82072914), and the Seed fund of the Second Hospital of Shandong University (S2015010015).

\section{Footnote}

Reporting Checklist: The authors have completed the STROBE reporting checklist. Available at http://dx.doi. org/10.21037/tbcr-21-1

Data Sharing Statement: Available at http://dx.doi. org/10.21037/tbcr-21-1

Peer Review File: Available at http://dx.doi.org/10.21037/ tbcr-21-1

Conflicts of Interest: All authors have completed the ICMJE uniform disclosure form (available at http://dx.doi. org/10.21037/tbcr-21-1). ZGY serves as an unpaid editorial board of Translational Breast Cancer Research. The other authors have no conflicts of interest to declare.

Ethical Statement: The authors are accountable for all aspects of the work in ensuring that questions related to the accuracy or integrity of any part of the work are appropriately investigated and resolved. This study was conducted in accordance with the Declaration of Helsinki (as revised in 2013) and the Harmonized Tripartite Guideline for Good Clinical Practice from the International Conference on Harmonization. This study was approved by Institutional Review Board of the Second Hospital of Shandong University (KYLL-2018(KJ)P-0019). Informed consent was obtained from all individual participants 
included in the study.

Open Access Statement: This is an Open Access article distributed in accordance with the Creative Commons Attribution-NonCommercial-NoDerivs 4.0 International License (CC BY-NC-ND 4.0), which permits the noncommercial replication and distribution of the article with the strict proviso that no changes or edits are made and the original work is properly cited (including links to both the formal publication through the relevant DOI and the license). See: https://creativecommons.org/licenses/by-nc-nd/4.0/.

\section{References}

1. Bray F, Ferlay J, Soerjomataram I, et al. Global cancer statistics 2018: GLOBOCAN estimates of incidence and mortality worldwide for 36 cancers in 185 countries. CA Cancer J Clin 2018;68:394-424.

2. Gunnarsdottir FB, Auoja N, Bendahl PO, et al. Colocalization of CD169 + macrophages and cancer cells in lymph node metastases of breast cancer patients is linked to improved prognosis and PDL1 expression. OncoImmunology 2020;9:1848067.

3. Lee SB, Kim HK, Choi Y, et al. Dynamic and subtypespecific interactions between tumour burden and prognosis in breast cancer. Sci Rep 2020;10:15445.

4. Lawn AM, Frampton AE, Krell J, et al. Lymph node ratio can further stratify prognosis in subpopulations of breast cancer patients with axillary nodal metastases. Future Oncol 2013;9:1425-31.

5. Vinh-Hung V, Nguyen NP, Cserni G, et al. Prognostic value of nodal ratios in node-positive breast cancer: a compiled update. Future Oncol 2009;5:1585-603.

6. Lai J, Pan Z, Deng H, et al. Personalized prognostic model incorporating axillary lymph node ratio and molecular subtype for predicting long-term survival in node-positive patients with breast cancer: A large-scale, multicenter study. Ann Oncol 2018;29:IX1.

7. Lai J, Pan Z, Chen P, et al. Development and validation of a nomogram incorporating axillary lymph node ratio to predict survival in node-positive breast cancer patients after neoadjuvant chemotherapy. Jpn J Clin Oncol 2019;49:22-8.

8. Kim SW, Choi DH, Huh SJ, et al. Lymph Node Ratio as a Risk Factor for Locoregional Recurrence in Breast Cancer Patients with 10 or More Axillary Nodes. Journal of Breast Cancer 2016;19:169-75.

9. Alam SM, Clark JS, George WD, et al. Altered lymphocyte populations in tumour invaded nodes of breast cancer patients. Immunol Lett 1993;35:229-34.

10. Kuroda H, Jamiyan T, Yamaguchi R, et al. Tumorinfiltrating B cells and $\mathrm{T}$ cells correlate with postoperative prognosis in triple-negative carcinoma of the breast. BMC Cancer 2021;21:286.

11. Fontenot JD, Gavin MA, Rudensky AY. Foxp3 programs the development and function of CD4+CD25+ regulatory T cells. Nat Immunol 2003;4:330-6.

12. Bates GJ, Fox SB, Han C, et al. Quantification of regulatory $\mathrm{T}$ cells enables the identification of high-risk breast cancer patients and those at risk of late relapse. J Clin Oncol 2006;24:5373-80.

13. Yeong J, Thike AA, Lim JCT, et al. Higher densities of Foxp3+regulatory $\mathrm{T}$ cells are associated with better prognosis in triple-negative breast cancer. Breast Cancer Res Treat 2017;163:21-35.

14. Miyara M, Gorochov G, Ehrenstein M, et al. Human FoxP3 + regulatory T cells in systemic autoimmune diseases. Autoimmun Rev 2011;10:744-55.

15. Vadasz Z, Toubi E. FoxP3 Expression in Macrophages, Cancer, and B Cells-Is It Real? Clin Rev Allergy Immunol 2017;52:364-72.

16. Zhao X, Ding L, Lu Z, et al. Diminished CD68+ CancerAssociated Fibroblast Subset Induces Regulatory T-Cell (Treg) Infiltration and Predicts Poor Prognosis of Oral Squamous Cell Carcinoma Patients. Am J Pathol 2020;190:886-99.

17. Hinz S, Pagerols-Raluy L, Oberg HH, et al. Foxp3 expression in pancreatic carcinoma cells as a novel mechanism of immune evasion in cancer. Cancer Res 2007;67:8344-50.

18. Takehara Y, Satoh T, Nishizawa A, et al. Anti-tumor effects of inactivated Sendai virus particles with an IL-2 gene on angiosarcoma. Clin Immunol 2013;149:1-10.

19. Kobayashi N, Kubota K, Kato S, et al. FOXP3+ regulatory $\mathrm{T}$ cells and tumoral indoleamine 2,3-dioxygenase expression predicts the carcinogenesis of intraductal papillary mucinous neoplasms of the pancreas. Pancreatology 2010;10:631-40.

20. Lal A, Chan L, Devries S, et al. FOXP3-positive regulatory $\mathrm{T}$ lymphocytes and epithelial FOXP3 expression in synchronous normal, ductal carcinoma in situ, and invasive cancer of the breast. Breast Cancer Res Treat 2013;139:381-90.

21. Shou J, Zhang Z, Lai Y, et al. Worse outcome in breast cancer with higher tumor-infiltrating FOXP3 + Tregs: a systematic review and meta-analysis. BMC Cancer 
2016;16:687.

22. Mahmoud SM, Paish EC, Powe DG, et al. An evaluation of the clinical significance of FOXP3 + infiltrating cells in human breast cancer. Breast Cancer Res Treat 2011;127:99-108.

23. Gökmen-Polar Y, Thorat MA, Sojitra P, et al. FOXP3 expression and nodal metastasis of breast cancer. Cell Oncol (Dordr) 2013;36:405-9.

24. Kawai H, Ishii A, Washiya K, et al. Estrogen receptor alpha and beta are prognostic factors in non-small cell lung cancer. Clin Cancer Res 2005;11:5084-9.

25. Xiang YJ, Guo MM, Zhou CJ, et al. Absence of gammainterferon-inducible lysosomal thiol reductase (GILT) is associated with poor disease-free survival in breast cancer patients. PLoS One 2014;9:e109449.

26. Lee S, Cho EY, Park YH, et al. Prognostic impact of FOXP3 expression in triple-negative breast cancer. Acta Oncol 2013;52:73-81.

27. Yoon HH, Orrock JM, Foster NR, et al. Prognostic impact of FoxP3 + regulatory $\mathrm{T}$ cells in relation to CD8+ T lymphocyte density in human colon carcinomas. PLoS One 2012;7:e42274.

28. Ladanyi A, Mohos A, Somlai B, et al. FOXP3+ cell density in primary tumor has no prognostic impact in patients with cutaneous malignant melanoma. Pathol Oncol Res 2010;16:303-9.

29. Sadahiro S, Suzuki T, Tanaka A, et al. Increase in tumor-infiltrating FoxP3-positive regulatory $\mathrm{T}$ cells in

doi: $10.21037 /$ tbcr-21-1

Cite this article as: Xiang YJ, Feng M, Liu LY, Wang F, Yu LX, Zhou F, Huang SY, Zheng C, Ye CM, Zhou WZ, Yin GS, Ma ZB, Li L, Zhang Q, Fu QY, Yu ZG. The role of FOXP3 of interstitial tissue in the pathogenesis of breast cancer. Transl Breast Cancer Res 2021;2:11. left-sided colorectal cancer tissues after preoperative oral uracil and tegafur/leucovorin chemotherapy. Ann Oncol 2018;29.

30. Niu J, Jiang C, Li C, et al. Foxp3 expression in melanoma cells as a possible mechanism of resistance to immune destruction. Cancer Immunol Immunother 2011;60:1109-18.

31. Xia M, Zhao MQ, Wu K, et al. Investigations on the clinical significance of FOXP3 protein expression in cervical oesophageal cancer and the number of FOXP3+ tumour-infiltrating lymphocytes. J Int Med Res 2013;41:1002-8.

32. Krausz LT, Fischer-Fodor E, Major ZZ, et al. GITRexpressing regulatory $\mathrm{T}$-cell subsets are increased in tumor-positive lymph nodes from advanced breast cancer patients as compared to tumor-negative lymph nodes. Int J Immunopathol Pharmacol 2012;25:59-66.

33. Nakamura R, Sakakibara M, Nagashima T, et al. Accumulation of regulatory $\mathrm{T}$ cells in sentinel lymph nodes is a prognostic predictor in patients with nodenegative breast cancer. Eur J Cancer 2009;45:2123-31.

34. Kapoor A, Vogel VG. Prognostic factors for breast cancer and their use in the clinical setting. Expert Rev Anticancer Ther 2005;5:269-81.

35. Frith K, Joly AL, Ma CS, et al. The FOXP3 $\Delta 2$ isoform supports Treg cell development and protects against severe IPEX syndrome. J Allergy Clin Immunol 2019;144:317-20.e8. 